\title{
Fuzzy Region Competition: A Convex Two-Phase Segmentation Framework
}

\author{
Benoit Mory and Roberto Ardon \\ Philips Medical Systems Research Paris, \\ 51 rue Carnot, B.P. 301, F-92156 SURESNES Cedex, FRANCE \\ \{benoit.mory, roberto.ardon\}@philips.com
}

\begin{abstract}
We describe a novel framework for two-phase image segmentation, namely the Fuzzy Region Competition. The functional involved in several existing models related to the idea of Region Competition is extended by the introduction of a fuzzy membership function. The new problem is convex and the set of its global solutions turns out to be stable under thresholding, operation that also provides solutions to the corresponding classical formulations. The advantages are then shown in the piecewise-constant case. Finally, motivated by medical applications such as angiography, we derive a fast algorithm for segmenting images into two non-overlapping smooth regions. Compared to existing piecewise-smooth approaches, this last model has the unique advantage of featuring closedform solutions for the approximation functions in each region based on normalized convolutions. Results are shown on synthetic 2D images and real $3 \mathrm{D}$ volumes.
\end{abstract}

\section{Introduction}

Several successful variational approaches to region-based image segmentation are based on the minimization of a functional that includes both boundary and region integrals $[5,7,11,17]$. When a two-phase partition of an image $I$ is considered, a general form of the optimization problem reads :

$$
\left(\mathcal{P}_{0}\right) \min _{\substack{\Sigma \subset \Omega \\ \boldsymbol{\alpha} \in A}}\left\{F_{0}(\Sigma, \boldsymbol{\alpha})=\int_{\Gamma} g(\Gamma(s)) d s+\int_{\Sigma} r_{1}^{\alpha_{1}}(x) d x+\int_{\Omega \backslash \Sigma} r_{2}^{\alpha_{2}}(x) d x\right\}
$$

where $\Omega \subset \mathbb{R}^{\mathrm{n}}$ is the image domain, $\Sigma \subset \Omega$ the foreground region and $\Gamma=\partial \Sigma$ its boundary. The error functions $r_{i}^{\alpha_{i}}: \Omega \rightarrow \mathbb{R}$ encode the underlying model of each region in terms of intensity properties. They may depend on a set of region parameters $\boldsymbol{\alpha}=\left(\alpha_{1}, \alpha_{2}\right)$, such as a couple of scalars [5], vectors [11,17] or functions $[13,14]$ (see Table 1$). g$ is a positive boundary potential, usually chosen to be a decreasing function of the image gradient. If the optimal $\boldsymbol{\alpha}$ is known $a$ priori, $\left(\mathcal{P}_{0}\right)$ is a supervised segmentation problem, constrained by the geodesic length of the boundary [11]. Otherwise, the segmentation is unsupervised and $\boldsymbol{\alpha}$ has to be included in the optimization process. A natural approach is then to perform successive minimization steps alternatively on $\Sigma$ (the partition variable) and on the components of $\boldsymbol{\alpha}$ (the region parameters). 
Table 1. Possible error functions $r_{i}$ and region parameters

\begin{tabular}{llr}
\hline Active Contours Without Edges [5] & $r_{i}=\lambda\left(I-c_{i}\right)^{2}$ & $A=\mathbb{R}^{2}$ \\
\hline $\begin{array}{l}\text { Region Competition [17] } \\
\text { Geodesic Active Regions [1]] }\end{array}$ & $r_{i}=-\lambda \log P_{i}\left(I \mid \alpha_{i}\right)$ & $A=\left(\mathbb{R}^{\mathrm{k}}\right)^{2}$ \\
\hline Mumford-Shah $[9,13,14]$ & $r_{i}=\lambda\left(I-s_{i}\right)^{2}+\mu\left|\nabla s_{i}\right|^{2}$ & $A=\left(\mathcal{C}^{1}\right)^{2}$ \\
\hline
\end{tabular}

Since the Region Competition ${ }^{1}$ algorithm [17], a popular way of solving $\left(\mathcal{P}_{0}\right)$ is to rewrite $F_{0}$ as a functional that only contains boundary integrals, using Green's theorem. Gradient-descent evolution schemes can then be built upon optimality conditions for $\Gamma$ (Euler-Lagrange equations). The boundary evolution is most often carried out numerically in the Level Sets framework [10] in which $\Gamma$ is represented implicitly by a real-valued function $\phi$ such that $\Gamma=\phi^{-1}(0)$. This boundary-oriented approach has intrinsic convergence limitations, since neither the involved functional nor the optimization space - the set of curves - are convex. In practice, this produces a dependency on initial conditions and prevents the use of powerful tools from convex optimization theory.

A different perspective on $\left(\mathcal{P}_{0}\right)$ is to rewrite $F_{0}$ as a functional that only contains integrals over the whole domain $\Omega$. To that end, Level Sets have also proved useful since the Active Contours Without Edges and related models [16, 5, 14]. The key idea is to replace in $F_{0}$ the set $\Sigma$ by the Heaviside function $H(\phi)$ and derive Euler-Lagrange equations directly for $\phi$, without explicit use of the boundary. This approach inherits some aforementioned drawbacks. It is ill-posed ${ }^{2}$ in $\phi$ and the optimization space - the set of characteristic functions - is still clearly non-convex. This recently motivated very promising works $[2,4]$ that consider alternative convex methods based on total variation regularization for the global minimization with respect to $\phi$.

Along the same line, our present contribution is three-fold. In section 2 , we first propose a generic formulation that has a number of interesting properties, including convexity with respect to the partition variable. This leads to a new framework, namely the Fuzzy Region Competition, that can be applied to any problem under the form $\left(\mathcal{P}_{0}\right)$. We illustrate the supervised case by using a statistical region term borrowed from the original Region Competition [17]. In section 3 , we also apply our convex framework to the classical error function of the cartoon Mumford-Shah functional [5,9], assuming constant regions. In section 4, motivated by medical applications such as angiography, we finally propose, develop and illustrate a new localized extension of this model that includes an intrinsic notion of scale, namely the Smooth Region Competition. Built on windowing and convolutions, this last segmentation method is very efficient and gives qualitative results that are similar to existing piecewise-smooth models based on Level Sets. Examples are shown in 2D and 3D.

\footnotetext{
${ }^{1}$ The name illustrates the competition between two repulsive forces applied at the boundary, depending on the competition function $r=r_{1}-r_{2}$.

${ }^{2}$ An infinite number of solutions $\phi$ are valid representations of a given optimal $\Sigma$.
} 


\section{Two-phase Fuzzy Region Competition}

The crux of our general formulation is that $\left(\mathcal{P}_{0}\right)$ can be solved by considering a closely related problem that is at least convex in its partition variable. Indeed, $\left(\mathcal{P}_{0}\right)$ is not convex since the set of sub-domains $\Sigma \subset \Omega$ is not convex. Nonetheless, it can be expressed as an optimization problem in the set of characteristic functions $^{3}$ (still non-convex) so that it also reads

$$
\min _{\chi, \boldsymbol{\alpha}}\left\{\tilde{F}_{0}(\chi, \boldsymbol{\alpha})=\int_{\Omega} g(x)|\nabla \chi(x)| d x+\int_{\Omega} \chi(x) r_{1}^{\alpha_{1}}(x) d x+\int_{\Omega}(1-\chi(x)) r_{2}^{\alpha_{2}}(x) d x\right\}
$$

Under this form, we propose to extend $\left(\mathcal{P}_{0}\right)$ into a problem that is convex in its partition variable, replacing the characteristic function $\chi$ by a fuzzy membership function $u$ belonging to a convex set. A suitable choice for this set is the space of functions of bounded variations taking their values in $[0,1]$, noted hereafter $B V_{[0,1]}(\Omega)$. This extension leads to the new Fuzzy Region Competition problem:

$$
(\mathcal{P}) \min _{u \in B V_{[0,1]}, \boldsymbol{\alpha}}\left\{F(u, \boldsymbol{\alpha})=\int_{\Omega} g|\nabla u|+\int_{\Omega} u(x) r_{1}^{\alpha_{1}}(x) d x+\int_{\Omega}(1-u(x)) r_{2}^{\alpha_{2}}(x) d x\right\}
$$

Being convex in $u$, problem $(\mathcal{P})$ has only solutions globally minimizing $F$ and can be solved (for fixed $\boldsymbol{\alpha}$ ) with efficient algorithms. Note that a multi-phase formulation with membership functions has recently been used with a different regularization term in [12] for 'soft' segmentation. Our formulation has the advantage of also providing solutions to the 'hard' segmentation problem $\left(\mathcal{P}_{0}\right)$ :

Proposition 1. Fixing $\boldsymbol{\alpha}$, if $u^{*}$ is a global minimizer of $F$ in $B V_{[0,1]}(\Omega)$ then for almost every $t \in[0,1]$, the characteristic function ${ }^{4} x \mapsto \chi_{u^{*}}(x, t)$ of the set $\Sigma_{t}=\left\{x \in \Omega, u^{*}(x)>t\right\}$ is also a global minimizer of $F$. In addition, $\Sigma_{t}$ is a global minimizer of $F_{0}$.

Proof: For any functions $u$ in $B V_{[0,1]}(\Omega)$ and $r$ in $L^{1}(\Omega)$, we have:

$$
\begin{gathered}
\text { Coarea formula }[6]: \quad \quad \int_{\Omega} g|\nabla u|=\int_{0}^{1} \int_{\Omega} g(x)\left|\nabla \chi_{u}(x, t)\right| d x d t \\
\int_{\Omega} u r=\int_{\Omega}\left(\int_{0}^{u(x)} d t\right) r(x) d x=\int_{\Omega}\left(\int_{0}^{1} \chi_{u}(x, t) d t\right) r(x) d x=\int_{0}^{1} \int_{\Omega} \chi_{u}(x, t) r(x) d x d t
\end{gathered}
$$

For sake of simplicity, we shall omit the fixed variable $\boldsymbol{\alpha}$ in the following. Applying (1) and (2) to the minimizer $u^{*}$, we obtain $F\left(u^{*}\right)=\int_{0}^{1} F\left(\chi_{u^{*}}(\cdot, t)\right) d t$, which is equivalent to $\int_{0}^{1}\left\{F\left(\chi_{u^{*}}(\cdot, t)\right)-F\left(u^{*}\right)\right\} d t=0$. Since $\forall t, F\left(u^{*}\right) \leqslant F\left(\chi_{u^{*}}(\cdot, t)\right)$, $F\left(\chi_{u^{*}}(\cdot, t)\right)=F\left(u^{*}\right)$ for a.e. $t \in[0,1]$. This means that the function $x \mapsto \chi_{u^{*}}(x, t)$ is also a minimizer of $F(\cdot, \boldsymbol{\alpha})$ for almost every $t \in[0,1]$. In addition, if $\Sigma \subset$ $\Omega$ is such that $F_{0}(\Sigma)<F\left(u^{*}\right)$ then its characteristic function ${ }^{3}$ also satisfies $F_{0}(\Sigma)=F\left(\chi^{\Sigma}\right)<F\left(u^{*}\right)$, which is a contradiction. Since for a.e $t \in[0,1] F_{0}\left(\Sigma_{t}\right)=$ $F\left(\chi_{u^{*}}(\cdot, t)\right)=F\left(u^{*}\right)$, we finally have $\forall \Sigma \subset \Omega, F_{0}\left(\Sigma_{t}\right) \leqslant F_{0}(\Sigma) . \square$

3 The characteristic function of a set $\Sigma \subset \Omega$ is the function $\chi^{\Sigma}(x)=1$ if $x \in \Sigma, 0$ otherwise. The perimeter of its boundary is given by $\operatorname{Per}(\partial \Sigma)=\int_{\Omega}|\nabla \chi|$.

${ }^{4} \chi_{u}$ is the function defined in $\Omega \times[0,1]$ by $\chi_{u}(x, t)=1$ if $u(x)>t, 0$ otherwise. 
In other words, we proved that the set of solutions of $(\mathcal{P})$ is stable under thresholding and it is clear that every solution of $\mathcal{P}_{0}$ corresponds to one of these thresholded functions. We can now develop an efficient optimization strategy capable of solving any two-phase segmentation problem that can be expressed through the general formulation $\left(\mathcal{P}_{0}\right)$, as the classical models listed in Table 1 . Indeed, being convex in $u$, problem $(\mathcal{P})$ may be solved through powerful tools from convex optimization theory. In the general unsupervised setting, $F$ can be minimized iteratively by alternating the following two steps:

(A) Considering $u$ fixed, optimize and update the region parameters $\boldsymbol{\alpha}$,
(B) Considering $\boldsymbol{\alpha}$ fixed, minimize w.r.t the partition variable and update $u$.
When a steady state $u^{*}$ is found, simply threshold it.

Step (A) depends on the specific choice of error functions (see sections 3 and 4). Step (B), however, can be realized by applying a generic minimization scheme for the variable $u$, as in [2]: Fixing $\boldsymbol{\alpha}$, minimizing $F$ with respect to $u$ in $B V_{[0,1]}$ is equivalent to minimizing $\int_{\Omega} g|\nabla u|+\int_{\Omega} u r$ in $B V$ under the constraint $0 \leqslant u \leqslant 1$, where $r=r_{1}^{\alpha_{1}}-r_{2}^{\alpha_{2}}$ is the competition function. Based on [4], this constrained problem has the same set of minimizers than the unconstrained problem $(\tilde{\mathcal{P}})$ of minimizing $\tilde{F}(u)=\int_{\Omega} g|\nabla u|+\int_{\Omega} u r+\beta \nu(u)$, with the exact penalty term $\nu(\xi)=\max (0,|2 \xi-1|-1)$ and $\beta>\frac{1}{2}|r|_{\infty}$. Even though $(\tilde{\mathcal{P}})$ can be numerically solved using a gradient-descent scheme based on the Euler-Lagrange equation, no advantage would be taken of the convexity of $\tilde{F}$. We thus choose to follow [2] and exploit the fast duality projection algorithm of Chambolle [3]. To that end, we add an auxiliary variable $v$ and consider the following weak approximation:

$$
\min _{(u, v) \in B V}\left\{\tilde{F}_{\theta}(u, v)=\int_{\Omega} g|\nabla u|+\frac{1}{2 \theta} \int_{\Omega}|u-v|^{2}+\int_{\Omega} r v+\beta \nu(v)\right\}
$$

where $\theta$ is chosen to be small enough so that the two components of any minimizing couple $\left(u^{*}, v^{*}\right)$ are almost identical w.r.t. the $L^{2}$ norm. Note that this approximation is still componentwise convex in $u$ and $v$. Moreover, $u$ being fixed, it is easy to check that the optimal $v$ is directly given by $v=\max (\min (u-\theta r, 1), 0))$. Now, $v$ being fixed, the projection algorithm of [3] can be applied for the minimization of $\tilde{F}_{\theta}$ with respect to $u$. Hence, $u=v-\theta \operatorname{div}(\boldsymbol{p})$ where the vector field $\boldsymbol{p}$ can be computed by using a fixed point algorithm, iterating on $n \geqslant 0$

$$
\text { taking } \tau>0, \quad \boldsymbol{p}^{0}=\mathbf{0}, \quad \boldsymbol{p}^{n+1}=\frac{\boldsymbol{p}^{n}+\tau \nabla\left(\operatorname{div}\left(\boldsymbol{p}^{n}\right)-v / \theta\right)}{1+\tau\left|\nabla\left(\operatorname{div}\left(\boldsymbol{p}^{n}\right)-v / \theta\right)\right| / g} .
$$

In practice, this algorithm is numerically very stable. Note that contrary to gradient-descent schemes, it does not rely on the explicit computation of the curvature of $u$. We also observed that decoupling $u$ and $v$ has a relaxation effect on the regularization and a very positive impact on the overall convergence.

In figure 1, we show an immediate application of the above generic scheme in a supervised segmentation experiment. The competition function $r=r_{1}-r_{2}$ has been built a priori, following the statistical model given in [11] or [17]. Probability distributions $P_{i}(I)$ have been estimated from both background and 


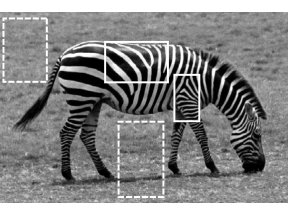

(a)
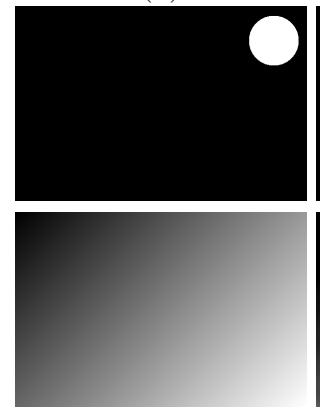

(e)

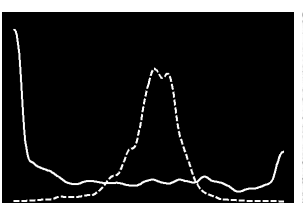

(b)
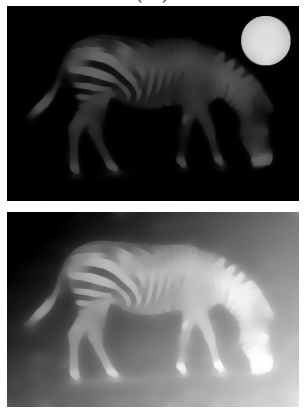

(f)

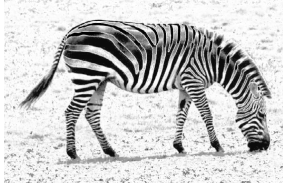

(c)

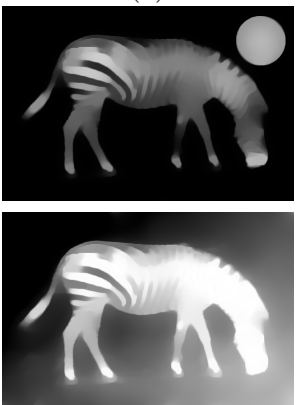

$(\mathrm{g})$

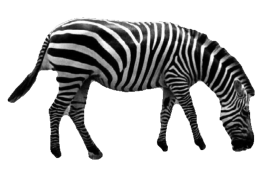

(d)
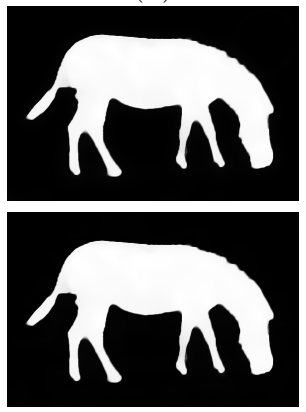

(h)

Fig. 1. Fuzzy Region Competition, supervised segmentation of the zebra image: (a) The original image and patches used to learn the background (dashed) and foreground (solid) histograms given in (b). (c) The corresponding competition function $r=\log \left(P_{2} / P_{1}\right)$. (d) Final segmentation. Two different (e) initializations, (f)-(g) intermediary states (step 10-20) and (h) final partition function $u$ (step 300).

foreground region patches, such that $r=\lambda \log \left(P_{2} / P_{1}\right)$, where $\lambda$ is a regularization parameter. In practice, we have observed that this supervised method gives always the same binary partition function, regardless of initial conditions. However, there exist 'pathological' cases of $r$ for which several minimizers of $F$, not necessarily binary, could solve the non strictly convex problem $(\mathcal{P})$. Those cases do not contradict proposition 1 , neither do they easily show up in practice, but it may be interesting to characterize them in a more in-depth study. In the following sections, we apply the Fuzzy Region Competition to some unsupervised cases, by first considering the classical two-phase constant model (section 3), then extending it to a new smooth model (section 4).

\section{Constant Region Competition}

In [4], Chan et al. propose to solve the minimization involved in the Active Contours Without Edges [5] by considering an auxiliary convex problem. They show that if $c_{1}$ and $c_{2}$ are fixed and $u^{*}$ is a solution of

$$
\min _{u \in B V_{[0,1]}(\Omega)}\left\{F_{[4]}(u)=\int_{\Omega}|\nabla u|+\lambda \int_{\Omega} u\left(\left(I-c_{1}\right)^{2}-\left(I-c_{2}\right)^{2}\right)\right\},
$$

then the set $\Sigma_{t}=\left\{x \in \Omega, u^{*}(x)>t\right\}$ is for a.e. $t \in[0,1]$ a minimizer of the following functional, considered in [5]:

$$
F_{[5]}(\Sigma)=\int_{\partial \Sigma} d s+\lambda \int_{\Sigma}\left(I-c_{1}\right)^{2}+\lambda \int_{\Omega \backslash \Sigma}\left(I-c_{2}\right)^{2}
$$


This result inspired the general formulation of section 2, and also guides its first application. Solving the Active Contours Without Edges model using our framework boils down to choosing $r_{i}^{c_{i}}=\lambda\left(I-c_{i}\right)^{2}, \boldsymbol{\alpha}=\left(c_{1}, c_{2}\right)$ and $g=1$ in $(\mathcal{P})^{5}$, such that the problem becomes

$$
\left(\mathcal{P}_{c}\right) \min _{\substack{u \in B V_{[0,1]} \\\left(c_{1}, c_{2}\right) \in \mathbb{R}^{2}}}\left\{F_{c}\left(u, c_{1}, c_{2}\right)=\int_{\Omega}|\nabla u|+\lambda \int_{\Omega} u\left(I-c_{1}\right)^{2}+\lambda \int_{\Omega}(1-u)\left(I-c_{2}\right)^{2}\right\}
$$

where $\lambda$ is a parameter balancing the region error terms and the Total Variation regularization. Applied to $\left(\mathcal{P}_{c}\right)$, Proposition 1 gives the result already obtained in [4]. Nonetheless, although closely related by $F_{c}\left(u, c_{1}, c_{2}\right)=F_{[4]}\left(u, c_{1}, c_{2}\right)+\int_{\Omega}(I-$ $\left.c_{2}\right)^{2}$, the two involved functionals lead to a slightly different perspective. $F_{[4]}$ is not to be considered as a minimization on the triplet $\left(u, c_{1}, c_{2}\right)$ but as a convex alternative to obtain a minimizer of the original problem [5], when $c_{1}$ and $c_{2}$ are fixed. Indeed, no optimization of those constants is involved in [4]. In practice, this implies the choice of an arbitrary level set of $u$ (e.g. 0.5) in step (A) of the alternate minimization scheme. This may at first seem a minor issue since any $\Sigma_{t}$ should be a minimizer at convergence. However, full convergence of $u$ is rarely obtained in practice at each step (B), in particular in the first iterations, and the levels of $u$ are not equivalent. Hence the arbitrary choice of a level $t$ for the computation of $c_{1}$ and $c_{2}$ may introduce a bias in the optimization process. On the contrary, in the proposed approach, the derivatives of $F_{c}$ with respect to scalars $c_{1}$ and $c_{2}$ give directly new optimality conditions that naturally involve all levels of the fuzzy membership function ${ }^{6} u$ :

$$
c_{1}^{*}=\frac{\int_{\Omega} u I}{\int_{\Omega} u}, \quad c_{2}^{*}=\frac{\int_{\Omega}(1-u) I}{\int_{\Omega}(1-u)}
$$

Our Constant Region Competition algorithm follows the generic scheme presented in section 2: (A) $u$ being fixed, compute the weighted averages $c_{1}$ and $c_{2}$ using equation (3). Then, (B) $c_{1}$ and $c_{2}$ being fixed, minimize w.r.t. $u$ using the weak formulation $\left(\widetilde{\mathcal{P}}_{\theta}\right)$ and perform a few iterations of Chambolle's projection algorithm. At convergence, the final segmentation is obtained by thresholding $u$ at any level in $[0,1]$, as justified by Proposition 1 . In figure 2 , we give an example of this simple unsupervised segmentation algorithm on a synthetic image. As in the supervised case, at least above a reasonable value of $\lambda$, we observed that the steady state is again always a binary function that is independent of the initialization. This method can thus be a useful tool for segmenting two-phase images corrupted by a gaussian noise of constant variance (the underlying assumption). Unfortunately, this is hardly realistic for medical images, in particular in angiography, since vessels usually exhibit a slowly varying contrast across the image (figure 3.a). In addition, extending the model in order to handle more than two phases would not necessarily be of any help if the observed variations are smooth. In the next section, we derive a new segmentation model by adding in the region

\footnotetext{
${ }^{5}$ For sake of simplicity, $g$ will be kept constant in the remainder (euclidian length).

${ }^{6}$ If $u$ is 0 almost everywhere (or 1 almost everywhere), $c_{1}^{*}=c_{2}^{*}=\int_{\Omega} I /|\Omega|$.
} 


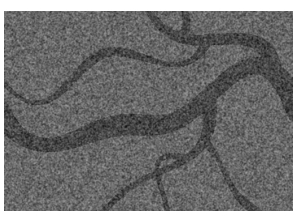

(a)
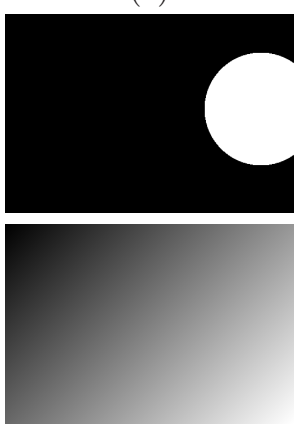

(e)

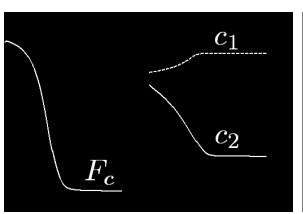

(b)
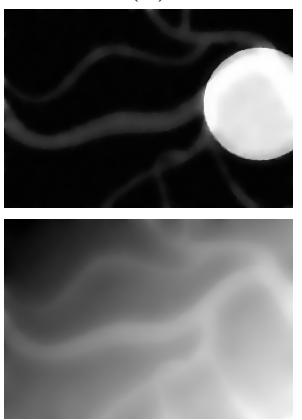

(f)

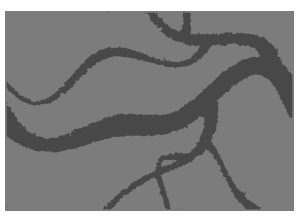

(c)
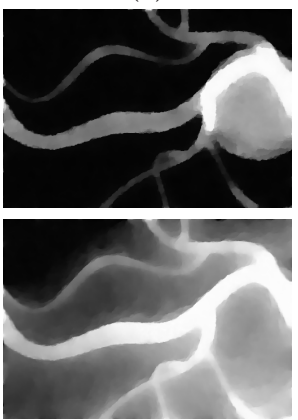

$(\mathrm{g})$

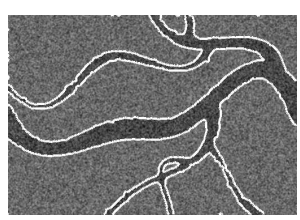

(d)
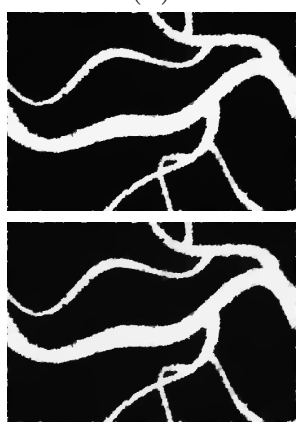

(h)

Fig. 2. Constant Region Competition : (a) A noisy synthetic angiography image where only two intensity means are present. (b) Convergence plots for $F_{c}, c_{1}$ and $c_{2}$. (c) Final approximation $u c_{1}+(1-u) c_{2}$. (d) Final segmentation. Two different (e) initial conditions, (f)-(g) intermediary steps and (h) final partition functions $u$.

error a localizing window, which explicitly provides an intrinsic notion of scale to our two-phase separation problem.

\section{Smooth Region Competition}

Even though two-phase images that could be accurately approximated by a single constant in each region are rarely encountered in practice, there are certainly some applications for which the hypothesis may still hold locally, i.e. at a certain scale. To that end, our purpose is to formulate a local extension of the previous model that would account for slowly varying intensities and still keep the advantages of the convex general framework $(\mathcal{P})$. A possible way to achieve this localization is to introduce a symmetrical window function $\omega: \Omega \mapsto \mathbb{R}^{+}$, such that $\omega(x) \rightarrow 0$ when $|x| \rightarrow+\infty$, and use it to design a continuous sum of local Constant Region Competitions. In the previous section, the global contribution of the foreground to the total error was $E=\int_{\Omega} u\left(I-c_{1}\right)^{2}$. Now windowing this error with $\omega$ in a neighborhood of $y \in \Omega$ gives :

$$
e(y)=\int_{x \in \Omega} u(x)\left(I(x)-s_{1}(y)\right)^{2} \omega(x-y) d x
$$

where the previously constant approximation $c_{1}$ is now an integrable function $s_{1}(y)$ that is allowed to vary in space. Hence, the new contribution of the foreground to the total error is $\int_{y \in \Omega} e(y) d y$. Adding a similar background contribution and switching the order of integrations finally exhibits our new region 
error terms, which leads to the Smooth Region Competition problem:

$\left(\mathcal{P}_{s}\right) \min _{\substack{u \in B V_{[0,1]} \\\left(s_{1}, s_{2}\right) \in L^{1}}}\left\{\begin{array}{r}F_{s}\left(u, s_{1}, s_{2}\right)=\int_{\Omega}|\nabla u|+\lambda \int_{x \in \Omega} u(x) \iint_{y \in \Omega} \omega(x-y)\left(I(x)-s_{1}(y)\right)^{2} d y d x \\ +\lambda \int_{x \in \Omega}(1-u(x)) \int_{y \in \Omega} \omega(x-y)\left(I(x)-s_{2}(y)\right)^{2} d y d x\end{array}\right.$

where $\lambda$ is a parameter balancing the error terms and the Total Variation regularization. $\left(\mathcal{P}_{s}\right)$ corresponds to the specific choices $\boldsymbol{\alpha}=\left(s_{1}, s_{2}\right), A=L^{1}(\Omega)^{2}$ and $r_{i}^{s_{i}}: x \rightarrow \lambda \int_{y \in \Omega} \omega(x-y)\left(I(x)-s_{i}(y)\right)^{2} d y$ in the general problem $(\mathcal{P})$, such that the generic scheme given in section 2 and Proposition 1 are still valid. Therefore, we only need to specify how to minimize with respect to $s_{1}$ and $s_{2}$.

Proposition 2. Fixing the partition variable $u$, the couple of functions $\left(s_{1}^{*}, s_{2}^{*}\right)$ that minimize $F_{s}\left(u, s_{1}, s_{2}\right)$ satisfy for almost every $y \in \Omega$ the following equations:

$$
\begin{aligned}
s_{1}^{*}(y) \int_{\Omega} u(x) \omega(x-y) d x-\int_{\Omega} u(x) I(x) \omega(x-y) d x & =0 \\
s_{2}^{*}(y) \int_{\Omega}(1-u(x)) \omega(x-y) d x-\int_{\Omega}(1-u(x)) I(x) \omega(x-y) d x & =0
\end{aligned}
$$

Consequently, as soon as there is a measurable set $M_{0}$ (resp. $M_{1}$ ) where $\forall x \in$ $M_{0}\left(\right.$ resp. $\left.M_{1}\right), u(x) \neq 0$ (resp. $u(x) \neq 1$ ) and if $\omega$ is a positive function, optimal closed-form solutions are given by the following normalized convolutions ${ }^{7}$ :

$$
s_{1}^{*}=\frac{\omega *(u I)}{\omega * u} \quad \text { and } \quad s_{2}^{*}=\frac{\omega *((1-u) I)}{\omega *(1-u)}
$$

Proof: With $f_{\eta}(t)=F_{s}\left(u, s_{1}+t \eta, s_{2}\right)$, the functional derivative of $F_{s}$ with respect to $s_{1}$ in the direction $\eta$ (a compactly supported $\mathcal{C}^{\infty}$ function) is given by

$$
\begin{aligned}
\left.\frac{d f_{\eta}}{d t}\right|_{t=0} & =-2 \lambda \int_{x \in \Omega} u(x) \int_{y \in \Omega}\left(I(x)-s_{1}(y)\right) \eta(y) \omega(x-y) d y d x \\
& =-2 \lambda \int_{y \in \Omega} \eta(y) \int_{x \in \Omega}\left(I(x)-s_{1}(y)\right) u(x) \omega(x-y) d x d y
\end{aligned}
$$

A necessary condition for $s_{1}^{*}$ to be a minimizer of $F_{s}$ is : $\forall \eta, d f_{\eta} /\left.d t\right|_{t=0}=0$. Thus $s_{1}^{*}$ must satisfy for almost every $y \in \Omega: \int_{x \in \Omega}\left(I(x)-s_{1}^{*}(y)\right) u(x) \omega(x-y) d x=0$. Similar derivations with respect to $s_{2}$ complete the proof.

The smooth case can be interpreted by analogy to the constant case of section 3 , where the weighted averages $c_{1}$ and $c_{2}$ have been replaced by the normalized convolutions $s_{1}$ and $s_{2}$. They should be considered as smooth approximations of the image within each fuzzy region. Indeed, even though no regularity constraint is explicit in $F_{s}$, the resulting functions are as regular as the window $\omega$, provided that the latter is positive. We remind that the theory of normalized

\footnotetext{
7 The convolution between $f$ and $g$ is function $f * g: x \rightarrow \int_{\Omega} f(y) g(x-y) d y$.
} 
convolution, introduced in [8], is a simple and useful extension of convolution that accounts for uncertain or missing image samples. Here, normalized convolutions naturally appear to create smooth approximations for each phase, taking into account the fuzzy membership functions $u$ and $(1-u)$ as certainty measures.

Our Smooth Region Competition algorithm follows again the generic scheme presented in section 2: (A) $u$ being fixed, compute the normalized convolutions $s_{1}$ and $s_{2}$ using equation (4). Then, (B) $s_{1}$ and $s_{2}$ being fixed, compute the competition function $r=r_{1}^{s_{1}}-r_{2}^{s_{2}}$ (this also involves convolutions ${ }^{8}$ ) and perform a few iterations of the dual projection algorithm . When a steady state is found, the final segmentation is obtained by thresholding $u$ at any level in $[0,1]$. The function $S=u s_{1}+(1-u) s_{2}$ gives then a piecewise-smooth approximation of the original image (see figure 3.c). Indeed, a binary $u$ prevents the smoothing of $s_{1}$ and $s_{2}$ across the boundary. In this respect, our approach relates to segmentation methods assuming a piecewise-smooth underlying model, such as those based on the Mumford-Shah functional [9]. However, it also features an essential practical advantage. Indeed, for instance in $[13,14,1]$, the approximation functions $s_{i}$ are defined as solutions of second-order differential equations, only defined inside each phase ${ }^{9}$. Although theoretically sound, this has two drawbacks in practice. First, diffusion equations with conditions on the boundary of an arbitrary-shaped domain (the current contour/surface) must be solved at each step (A) of the alternate minimization scheme. This is tedious since the domain is moving between each iteration. Second, both approximation functions are only defined in their respective domain, but need to be extended in order to compute the competition function, at least in a local vicinity. The construction of the extension is arbitrary and needs the exact location of the boundary separating the phases at all times. For 3D volumes in particular, those steps involve quite heavy computations. In contrast, step (A) of the Smooth Region Competition algorithm is much lighter since smooth closed-form solutions for $s_{1}$ and $s_{2}$, defined everywhere in $\Omega$, are directly constructed from $u$.

A very suitable choice for the window function is the normalized isotropic Gaussian kernel $\omega(x)=\left(2 \pi \sigma^{2}\right)^{-n / 2} \exp \left(-|x|^{2} / 2 \sigma^{2}\right)$. The standard deviation $\sigma$ explicitly provides the model with an intrinsic notion of scale, related to the intensity variations that are expected to arise in each region. Note that when $\sigma \rightarrow \infty$, we recover the constant model. Furthermore, being positive, non-compactly supported and $\mathcal{C}^{\infty}$, the Gaussian window guarantees the regularity of $s_{i}$ functions everywhere in the domain $\Omega$. As for the implementation, very efficient recursive filters that approximate the convolution and simulate the non-compact support with a computation cost that is independent on $\sigma$ are also available [15]. In figures 3 and 4 , we show the application of this algorithm on a $2 \mathrm{D}$ synthetic image and on real $3 \mathrm{D}$ volumes.

\footnotetext{
8 The competition function here reads $r(x)=\left[\omega *\left(s_{1}^{2}-s_{2}^{2}\right)\right](x)-2 I(x)\left[\omega *\left(s_{1}-s_{2}\right)\right](x)$. $9 \int_{\Sigma}\left|\nabla s_{1}\right|^{2}+\int_{\Omega \backslash \Sigma}\left|\nabla s_{2}\right|^{2}$ is typically added in the functional.
} 


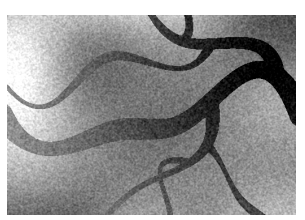

(a)
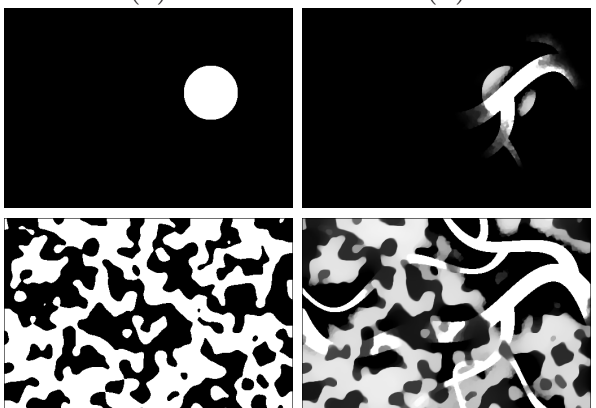

(e)

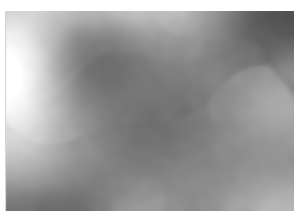

(b)

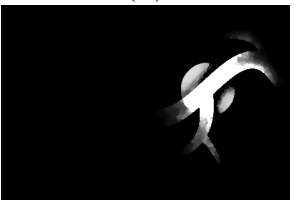

(f)

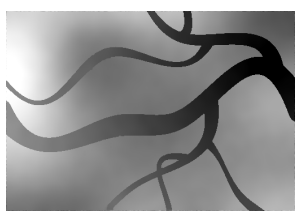

(c)
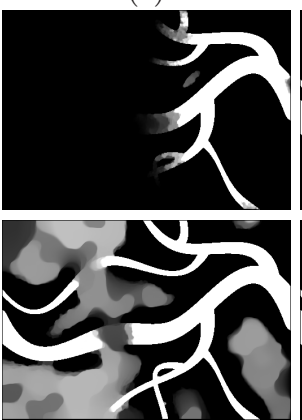

(g)

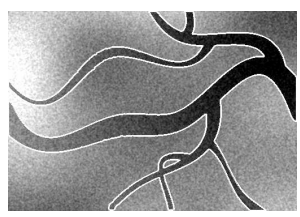

(d)
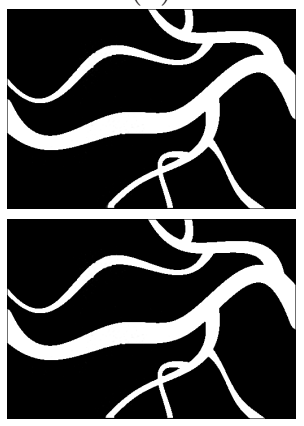

(h)

Fig. 3. Smooth Region Competition: (a) A noisy synthetic image of a vessel of smoothlyvarying intensity on a smooth background. (b) Final $s_{2}$. (c) Final smooth approximation $u s_{1}+(1-u) s_{2}$. (d) Segmentation. Two different (e) initial conditions, (f)-(g) intermediary steps and (h) final partition functions $u$. The circle radius in (e) is $4 \sigma$.

\section{Conclusion}

We introduced a new framework for two-phase image segmentation, namely the Fuzzy Region Competition. It generalizes some existing supervised and unsupervised region-based models, if only two phases are considered, and can be used in all applications where a distinction foreground / background is meaningful, in $2 \mathrm{D}$ or $3 \mathrm{D}$. The functional formulation we proposed is convex in its bounded variation partition variable $u$. The convexity guarantees to obtain in the supervised case a globally optimal solution. Although this property does not hold in the unsupervised setting, for which the region parameters have also to be optimized, it still produces very stable algorithms that turn out to be weakly sensitive to initial conditions. Furthermore, those algorithms can be very fast, in particular by using convex optimization tools and recent developments in total variation regularization. We believe that the use of a fuzzy membership function in regionbased segmentation models leads to easy implementations that are more efficient and stable than their existing curve evolution Level Sets counterparts. This motivates the need for further work, in particular by including some a priori shape knowledge within the model in order to increase robustness.

Based on this framework, a new consistent model for the partition of an image into two smooth components was also derived. It is extensively built around windowing principles, convolutions, and features closed-form approximations in each region. Thus, it can be faster than some of the existing variational methods that give qualitatively similar results. It also provides an intrinsic notion of 

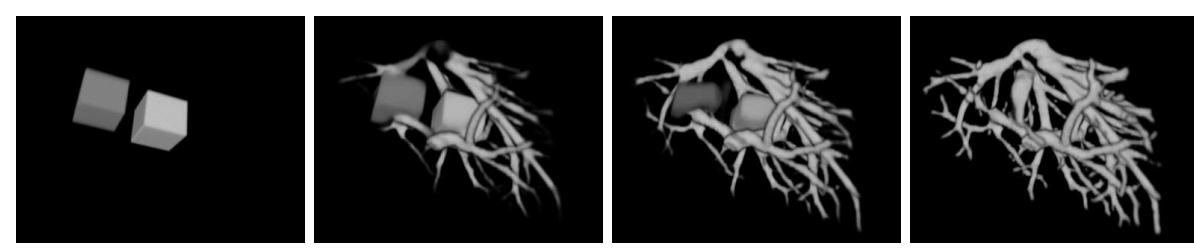

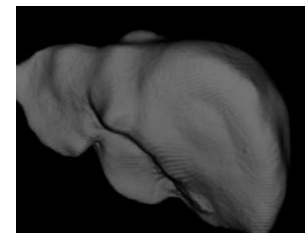

(a)

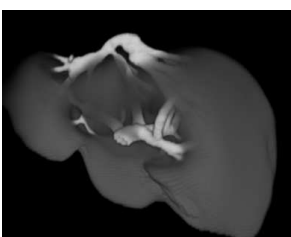

(b)

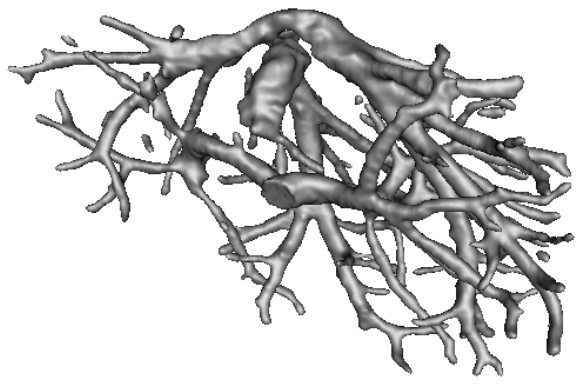

(e)

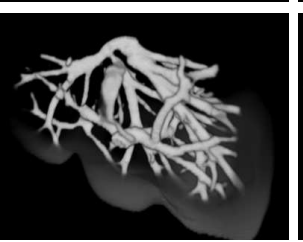

(c)

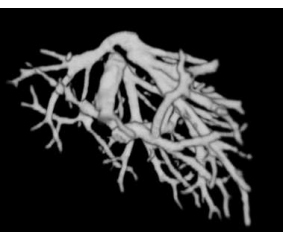

(d)

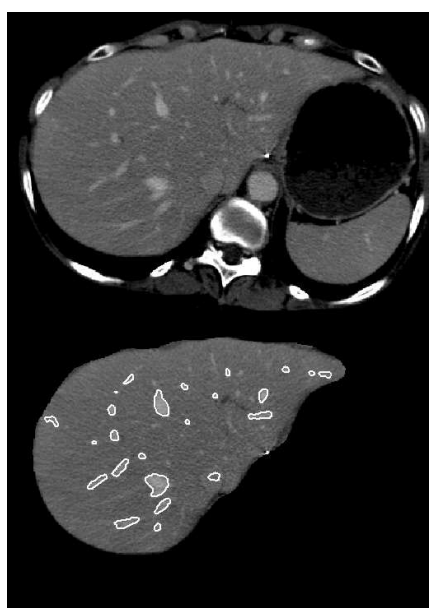

(f)

Fig. 4. A typical example of the Smooth Region Competition applied to medical images in 3D. We applied our model to the segmentation of a liver vessel tree from a 294x215x101 CT angiography. In this experiment, a segmentation mask of the liver was available, such that the two-phase hypothesis, (vessels + liver tissue), was approximately valid. In (a),(b),(c),(d), two evolutions of the fuzzy partition function $u$ are shown in volume rendering for different initializations. Note that at convergence, the result is almost binary and independent from the initial conditions. Computation time was about $20 \mathrm{sec}$ on a standard PC platform. (e) shows the resulting mesh in surface rendering after the final thresholding. (f) shows a slice of the original volume and the contours of final vessel segmentation overlaid on the masked liver.

scale, depending on the intensity variations expected to arise in the objects to be segmented. However, from a segmentation perspective, scale also intuitively relates to other geometrical quantities such as the smoothness of the boundary or the allowed size of foreground objects. Both are presently dependent on the chosen balance between the error terms and the TV regularization. This would probably need also to be better understood and studied in the future. 
Acknowledgments We would like to thank Dr X. Bresson, Dr O. Cuisenaire and Prof. J.P. Thiran for many inspiring discussions.

\section{References}

1. X. Bresson, S. Esedoglu, P. Vandergheynst, J.-P. Thiran, and S. Osher. Global minimizers of the active contour/snake model. No CAM Report 0504, UCLA, Dept. of Mathematics, 2005.

2. X. Bresson, S. Esedoglu, P. Vandergheynst, J.-P. Thiran, and S. Osher. Fast global minimization of the active contour/snake model. to appear in Journal of Mathematical Imaging and Vision, 2006.

3. A. Chambolle. An algorithm for total variation minimization and applications. Journal of Mathematical Imaging and Vision, 20(1-2):89-97, 2004.

4. T.F. Chan, S. Esedoglu, and M. Nikolova. Algorithms for finding global minimizers of image segmentation and denoising models. UCLA CAM Report 04-54, 2004.

5. T.F. Chan and L.A. Vese. Active contours without edges. IEEE Trans. on Image Processing, 10(2):266-277, February 2001.

6. W. Fleming and R. Rishel. An integral formula for total gradient variation. Archiv Der Mathematik, 11:218-222, 1960.

7. S. Jehan-Besson, M. Barlaud, and G. Aubert. Dream2s: Deformable regions driven by an eulerian accurate minimization method for image and video segmentation. Int. Journal of Computer Vision, 53(1):45-70, 2003.

8. H. Knutsson and C.F. Westin. Normalized and differential convolution: Methods for interpolation and filtering of incomplete and uncertain data. In Proc. of Computer Vision and Pattern Recognition, pages 515-523, New York City, USA, June 16-19 1993.

9. D. Mumford and J. Shah. Optimal approximations by piecewise smooth functions and associated variational problems. Comm. on Pure and Applied Mathematics, 42(5):577-685, 1989.

10. S. Osher and J.A. Sethian. Fronts propagating with curvature dependent speed: algorithms based on the hamilton-jacobi formulation. Journal of Computational Physics, 79:12-49, 1988.

11. N. Paragios and N. Deriche. Geodesic active regions and level set methods for supervised texture segmentation. Int. Journal of Computer Vision, 46(3):223-247, 2002.

12. Jianhong (Jackie) Shen. A stochastic-variational model for soft mumford-shah segmentation. International Journal of Biomedical Imaging, 2006(92329).

13. A. Tsai, A. Yezzi Jr., and A.S. Willsky. Curve evolution implementation of the mumford-shah functional for image segmentation, denoising, interpolation, and magnification. IEEE Trans. on Image Processing, 10(8):1169-1186, 2001.

14. L.A. Vese and T.F. Chan. A multiphase level set framework for image segmentation using the mumford and shah model. Int. Journal of Computer Vision, 50(3):271293, 2002.

15. I. Young and L. van Vliet. Recursive implementation of the gaussian filter. Signal Processing, 44:139-151, 1995.

16. H. K. Zhao, T. Chan, B. Merriman, and S. Osher. A variational level set approach to multiphase motion. Journal of Computational Physics, 127:179-195, 1996.

17. S. C. Zhu and A. Yuille. Region competition: Unifying snakes, region growing, and bayes/mdl for multiband image segmentation. IEEE Trans. On Pattern Analysis and Machine Intelligence, 18(9):884-900, September 1996. 